\title{
The association between social media addiction and eating disturbances is mediated by muscle dysmorphia-related symptoms: a cross-sectional study in a sample of young adults
}

\author{
Claudio Imperatori ${ }^{1} \cdot$ Angelo Panno $^{1}$. Giuseppe Alessio Carbone ${ }^{1} \cdot$ Ornella Corazza $^{2,3} \cdot$ Ines Taddei $^{4}$. \\ Laura Bernabei $^{4,5}$. Chiara Massullo ${ }^{1}$. Elisabeth Prevete ${ }^{4} \cdot$ Lorenzo Tarsitani $^{4} \cdot$ Massimo Pasquini $^{4}$. \\ Benedetto Farina ${ }^{1} \cdot$ Massimo Biondi $^{4} \cdot$ Francesco Saverio Bersani ${ }^{4}{ }^{4}$
}

Received: 11 April 2021 / Accepted: 28 May 2021 / Published online: 26 June 2021

(c) The Author(s) 2021

\begin{abstract}
Purpose Although the association between problematic use of the internet and eating disorders (EDs) in young adults has been previously established, its underlying mechanisms have not been completely clarified. It has been suggested that exposure to idealized very thin and toned body images (e.g., "thinspiration" and "fitspiration" trends) on social media might lead to increased feelings of body dissatisfaction which, in turn, can represent a trigger for EDs. We have tested this hypothesis in a sample $(N=721)$ of young adults (504 females, mean age: $24.13 \pm 3.70$ years; range $18-34)$.

Methods Self-report measures investigating symptoms related to social media addiction (SMA), muscle dysmorphia (MD), and EDs were used. A mediational model analyzing the direct and indirect effects of SMA-related symptoms on ED-related symptoms through the mediating role of MD-related symptoms was performed controlling for confounding factors (e.g., socio-demographic variables, substances use, body mass index, psychopathological distress).

Results The model showed that the total effect of SMA-related symptoms on ED-related symptoms was significant $(B=0.213$; $p=0.022)$ and that this association was mediated by MD-related symptoms $(B=0.083 ; p=0.021)$.

Discussion Our findings support the possibility that MD-related symptoms play a relevant role in mediating the association between SMA severity and ED pathology.

Level of evidence Level III, evidence obtained from well-designed cohort or case-control analytic studies.
\end{abstract}

Keywords Social media addiction $\cdot$ Eating disorders $\cdot$ Muscle dysmorphia $\cdot$ Substances use $\cdot$ Psychopathology

Francesco Saverio Bersani

francescosaverio.bersani@uniroma1.it

1 Cognitive and Clinical Psychology Laboratory, Department of Human Science, European University of Rome, Rome, Italy

2 Department of Clinical, Pharmaceutical and Biological Sciences, University of Hertfordshire, Hatfield, UK

3 Department of Medico-Surgical Sciences and Biotechnologies, Sapienza University of Rome, Latina, Italy

4 Department of Human Neurosciences, Sapienza University of Rome, Viale dell'Università 30, 00185 Rome, Italy

5 Mental Health Department, ASL Roma 5 Hospital, Rome, Italy

\section{Introduction}

The increasing relevance of the internet in everyone's life, and especially in the lives of adolescents and young adults, is raising interest on potential health-related sequelae associated with its use. In relation to mental health, recent studies have observed that dysfunctional and excessive use of internet, a condition often conceptualized as internet addiction or as problematic use of the internet (PUI) [1,2], is significantly related to mental health disturbances $[3,4]$.

A link between PUI and mental diseases has been consistently observed in relation to eating disorders (EDs) [5]. EDs are conditions characterized by disturbed attitudes towards weight, body image, and eating, which impair physical health and psychosocial functioning $[6,7]$. The Diagnostic and Statistical Manual of Mental Disorders 5th Edition (DSM5) includes a section on EDs titled "Feeding 
and Eating Disorders" which comprises the following diagnoses [7]: pica, rumination disorder (RD), avoidant/restrictive food intake disorder (ARFID), anorexia nervosa (AN), bulimia nervosa (BN), binge eating disorder (BED), other specified feeding or eating disorders (OSFEDs), and unspecified feeding or eating disorder (UFEDs). It is estimated that the lifetime prevalence of the main EDs (i.e., AN, BN, BED, OSFEDs) is about of $8 \%$ for women and $2 \%$ for men [8]. Furthermore, subthreshold EDs as well as ED-related symptoms (e.g., food preoccupation, binge eating, excessive dieting, body dissatisfaction, body image concerns, rigid weight-control behaviors) are widely detected with various degrees of severity in the general population, especially in young adults [9-12], as well as in patients with psychiatric diagnoses different from EDs [13-16].

In relation to the association between PUI and EDs, Hinojo-Lucena et al. recently performed two meta-analyses (involving data from over 8000 and over 5000 students) showing that both EDs (i.e., AN, BN, and BED) and EDrelated symptoms (i.e., food preoccupation, loss of control eating, and dieting) were significantly higher among individuals with PUI than in those without [5].

Although the association between PUI and ED-related symptoms in young adults has been observed consistently across studies, its underlying mechanisms have not been completely clarified. In fact, the construct of PUI includes heterogeneous internet-related phenomena: as an example, disturbances related to video gaming, social networking, pornography, cybersex, and online gambling, are all tightly related to PUI but can be characterized by different underlying psychological, behavioral and biological characteristics $[4,17]$.

In particular, it has been suggested that one of the possible underlying mechanisms of the association between PUI and EDs is the massive exposure to idealized stylized and toned body figures on social media (SM) [5]. Specifically, studies on emerging trends among young people have been suggesting that in SM (e.g., Facebook, Instagram), there are posts, often identified with the hashtag "thinspiration", which contribute to idealize extremely thin bodies, and that the exposure to such contents is associated with attitudes, symptoms and beliefs that characterize EDs [18-20]. Further, it has been suggested that the increasing amount of posts identified with the hashtag "fitspiration" (i.e., contents shared to promote healthy messages focused on fitness, exercise, eating styles, physical appearance, and weight control) can emphasize unrealistic body image as well as unattainable habits, thus leading to negative feelings in relation to mood, self-esteem, body image and emotional well-being [18, 21-25]. This can also contribute [26] to induce in vulnerable users symptoms related to a specific form of body dysmorphic disorder (BDD), the so called muscle dysmorphia (MD), characterized by extreme preoccupation over one's physical appearance and muscularity and by compulsive physical exercise [16, 27].

Taking this into consideration, it is possible that excessive use of SM leads to increased levels of body dissatisfaction and MD which, in turn, can represent triggers for ED-related pathology. In the present study, we aimed at cross-sectionally exploring this phenomenon and testing this hypothesis in a sample of young adults using assessment measures specifically designed to evaluate psychopathological symptoms related to SM use, EDs and MD. Specifically we conducted a mediation analysis to examine whether MD-related symptoms mediated the association between excessive SM use and ED-related symptoms, with potential confounding factors (socio-demographic variables, substances use/misuse, body mass index [BMI] and psychopathological distress) being controlled for. We hypothesized that excessive use of SM (i) is positively associated with both MD- and ED-related symptoms, and (ii) it is positively, significantly and indirectly related to ED-related symptoms through the severity of MD-related symptoms.

\section{Materials and methods}

\section{Participants and procedures}

According to the sample size guidelines of Fritz and MacKinnon [28], assuming small effect sizes for the " $a$ " and " $b$ " paths in the mediation model, the analyses required a minimum sample size of 558 with the bootstrapping procedure to provide a statistical power of 0.80 .

The present study involved 721 Italian young adults (504 females and 217 males; mean age: $24.13 \pm 3.70$ years; range 18-34). Due to the coronavirus disease-19 (COVID-19) outbreak, the recruitment was performed in two phases: (i) between November 2019 and March $2020(N=266,36.9 \%)$ through a convenience sampling approach using advertising material posted around traditional community groups and using self-administered paper/pencil questionnaires; (ii) between September 2020 and December $2020(N=455,63.1 \%)$ through an online survey shared using web-based tools (e.g., emails, mailing lists, SM, instant messaging).

All subjects voluntarily (i.e., they did not receive payment or other compensation) and anonymously participated to the present research and gave their written informed consent to take part to the study. Inclusion criteria were: (i) age between 18 and 34 years, (ii) good ability to understand written Italian, (iii) for the online survey, the correct response to one item of attentional quality check, and (iv) the provision of written consent. 


\section{Measures}

Information on socio-demographic and clinical variables [i.e., age, sex, body mass index (BMI, job status, education level, marital status, tobacco and illicit drugs use in the last 12 months] was collected. Subjects were asked to complete the following self-report measures: the Bergen Social Media Addiction Scale [BSMAS; 29], the Eating Attitudes Test-26 [EAT-26; 30], the Muscle Dysmorphic Disorder Inventory [MDDI; 31], the Brief Symptom Inventory [BSI; 32] and the Cut-Annoyed-Guilty-Eye (CAGE) questionnaire [33].

The BSMAS is a 6-item self-administered questionnaire assessing, in the last 12 months, addiction-like symptoms (i.e., salience, mood modification, tolerance, withdrawal, conflict, and relapse) in relation to excessive and compulsive use of SM (e.g., Facebook, Instagram, etc.). Items are rated on a 5-point Likert scale (from $1=$ "very rarely" to $5=$ "very often") with higher scores reflecting higher levels of symptoms related to social media addiction (SMA). A cut-off of $\geq 19$ has been used to screen problematic social media use (PSMU) [34]. In the present study the Italian version of the BSMAS [35] was used and the Cronbach's $\alpha$ was 0.85 .

The EAT-26 is a 26 -item self-report questionnaire commonly used to investigate symptoms and concerns characteristic of EDs [36], such as dieting and food preoccupation. Participants were asked to judge each items according to 6-point Likert scale (from "never" $=0$ to "always" $=3$ ) with total scores ranging from 0 to 78 . Higher scores reflect higher ED-related symptoms, and a cut-off of $\geq 20$ is widely used as the threshold value for clinically significant EDrelated pathology [37]. The Italian validated version of the EAT-26 used in this study has showed good psychometric properties [38]. Cronbach's alpha in our sample was 0.93 for the EAT-26 total score.

The MDDI is one of the most used tools for assessing MD [39]. It is a multi-dimensional self-report measure composed by 13 items and rated on a 5-point Likert-type scale (from $1=$ "never" to $5=$ "always") investigating three MD core dimensions, confirmed through factor analyses [39]: (i) drive for size (e.g., thoughts of being thinner and/or the desire to increase size and strength), (ii) appearance intolerance (e.g., negative beliefs about one's body), and (iii) functional impairment (e.g., excessive and compulsive exercise). A total score can be derived by the sum of the subscales. Higher values reflect higher MD-related symptoms, and a cut-off point of 39 has been proposed and used in previous reports to identify clinically relevant MD [40, 41]. Although the psychometric properties of the MDDI were most investigated in male samples, it has been recently reported [40] that the three-factor structure of the MDDI was independent of sex with no statistically significant difference in the MDDI total score. In the present study, the Italian version of the MDDI [42] was used and the Cronbach's $\alpha$ was 0.78 for the MDDI total score.

The BSI [32] is the short version of the Symptom Checklist-90R [43] and it is composed by 53 items rated on a 5-point Likert scale (0-4) assessing nine primary symptom dimensions: depression, somatization, obsessive-compulsive, interpersonal sensitivity, anxiety, hostility, phobic anxiety, paranoid ideation, and psychoticism. The BSI also provides a global severity index (GSI) which is designed to measure overall psychopathological distress. Higher scores indicate more severe self-reported symptoms. As recommended [32] and according to previous reports e.g., [44-46], in the present study the cutoff point of $63 \mathrm{~T}$ score on the GSI or in two primary dimensions was used to identify subjects with clinically relevant level of psychopathological distress. We used a previously validated Italian version of this scale [47] and the Cronbach's alpha in the present sample was 0.97 for the GSI.

The CAGE questionnaire is composed by 4 dichotomous $(0=$ "no"; $1=$ "yes") items and it is designed as screening tool for the detection of problematic alcohol use (PAU). The total score can range from 0 to 4 , with higher values reflecting more severe problematic patterns of alcohol use. A cut-off of $\geq 2$ is commonly used in the literature $[48,49]$ to screen PAU. In the present study, we used the Italian adaptation of the CAGE [33], and the Cronbach's $\alpha$ was 0.64 for the CAGE total score.

\section{Statistical analyses}

All analyses were performed using the SPSS (18.0) statistical package (IBM, Armonk, NY, USA). The association among variables was assessed using Spearman's $\rho$ correlation coefficients. For sensitivity analyses, differences between individuals with and without PSMU (i.e., BSMAS $\geq 19$ ) were also computed using two-way Chisquared $\left(\chi^{2}\right)$ and Mann-Whitney $U$ test for dichotomous and dimensional measures, respectively. Analysis-appropriate effect sizes (Cohen's $d$ and Cramer's $V$, respectively, for $U$ and $\chi^{2}$ tests) were computed [50] and converted to $r$ values [51]. Non-parametric tests were chosen as several of the considered variables were not normally distributed (Shapiro-Wilk test, $p<0.05$ ).

To determine whether the relationship between SMAand ED-related symptoms was mediated by MD severity, a mediation model using the Model 4 of the SPSS Macro Process [52] with 5000 bootstrap samples was performed. In the present study, we tested a model in which BSMAS total score (i.e., SMA-related symptoms) was the independent variable, EAT-26 total score (i.e., ED-related symptoms) was the dependent variable, and MDDI total score (MDrelated symptoms) was the mediator. Potentials cofounding 
sociodemographic (i.e., sex, age, educational level, marital and job status) and clinical (i.e., BMI, problematic alcohol use, tobacco and illicit drug use, and clinically relevant psychopathological distress) variables were also included in the model as covariates. Furthermore, due to the different recruitment method and taking into account the impact of COVID-19 pandemic on psychopathology (including potential impact on substance and behavioral addictions) [53-55], the compilation modality (i.e., paper/pencil vs online questionnaires) was also included as a covariate.

Although in the mediational models, the relations between variables are considered to be causal, for crosssectional studies, the mediation strategies should be considered as a type of variance partitioning, which can be useful when investigating whether the relation between two variables is reduced when a mediating variable is considered [56]. Thus, it is important to note that the adopted statistical design is correlational in nature, which precludes a definitive causal interpretation of the association between these variables. According to Baron and Kenny [57], we reported the results from different three models. In the first model (path a) we showed the direct effect of SMA-related symptoms on MD-related symptoms. In the second model (path $c^{\prime}$ ) we reported the direct effect of SMA-related symptoms on EDrelated symptoms. In the third and last model (path $c$ ), we showed the total effect, i.e., the sum of the direct and indirect effects, of SMA-related symptoms on ED-related symptoms.

\section{Ethics}

The present study was approved by the Ethics committee of the Department of Human Neurosciences of Sapienza University of Rome and was performed in accordance with the Helsinki Declaration criteria.

\section{Results}

In the present sample, there were 154 subjects (21.4\%) who met the criteria for PSMU, 120 (16.6\%) who met the criteria for clinically relevant ED-related symptoms, and 26 (3.6\%) who met the criteria for clinically relevant MD. Finally, there were 170 participants $(23.6 \%)$ who met the criteria for clinically relevant psychopathological distress, 94 (13.0\%) who met the criteria for PAU. Detailed clinical and socio-demographic characteristics of the sample are reported in Table 1.

SMA-related symptoms were positively associated with MD-related symptoms $(\rho=0.138 ; p<0.001)$, EDs-related symptoms $(\rho=0.179 ; p<0.001)$, psychopathological distress $(\rho=0.430 ; p<0.001)$ and PAU severity $(\rho=0.105$; $p=0.005$ ). A similar pattern of correlations was detected for both MDDI and EAT-26 total score. SMA-related symptoms were also negatively associated with both age $(\rho=-0.163$;
Table 1 Descriptive statistics for the sample $(N=721)$

\begin{tabular}{ll}
\hline Variables & \\
\hline Age, $\mathrm{M} \pm \mathrm{SD}$ & $24.13 \pm 3.70$ \\
Females, $N(\%)$ & $504(69.9)$ \\
Occupation & \\
Employed, $N(\%)$ & $195(27.1)$ \\
Unemployed, $N(\%)$ & $47(6.5)$ \\
Students, $N(\%)$ & $479(66.4)$ \\
Married or living with partner, $N(\%)$ & $84(11.7)$ \\
Educational level $>13$ years, $N(\%)$ & $316(43.8)$ \\
Tobacco use in the last $12 \mathrm{months}, N(\%)$ & $311(43.1)$ \\
Substance use in the last $12 \mathrm{months}, N(\%)$ & $161(22.3)$ \\
CAGE total score, $\mathrm{M} \pm \mathrm{SD}$ & $0.45 \pm 0.86$ \\
CAGE $\geq 2, N(\%)$ & $94(13.0)$ \\
Self-reported $\mathrm{BMI}, \mathrm{M} \pm \mathrm{SD}$ & $22.10 \pm 3.28$ \\
BMI $<18.5 \mathrm{~kg} / \mathrm{m}^{2}, N(\%)$ & $77(10.7)$ \\
BMI between 18.50 and $24.99 \mathrm{~kg} / \mathrm{m}^{2}, N(\%)$ & $534(74.1)$ \\
BMI between 25 and $29.9 \mathrm{~kg} / \mathrm{m}^{2}, N(\%)$ & $95(13.2)$ \\
BMI $\geq 30 \mathrm{~kg} / \mathrm{m}^{2}, N(\%)$ & $15(2.1)$ \\
BSMAS total score, $\mathrm{M} \pm \mathrm{SD}$ & $14.21 \pm 5.47$ \\
BSMAS $\geq 19, N(\%)$ & $154(21.4)$ \\
EAT-26 total score, $\mathrm{M} \pm \mathrm{SD}$ & $10.08 \pm 12.93$ \\
EAT-26 $\geq 20, N(\%)$ & $120(16.6)$ \\
MDDI total score, $\mathrm{M} \pm \mathrm{SD}$ & $23.33 \pm 7.65$ \\
MDDI $>39, N(\%)$ & $26(3.6)$ \\
BSI-GSI, $\mathrm{M} \pm \mathrm{SD}$ & $0.97 \pm 0.70$ \\
BSI cut-off** $N(\%)$ & $170(23.6)$ \\
\hline
\end{tabular}

$M$ mean, $S D$ standard deviation, $C A G E$ Cut-Annoyed-Guilty-Eye (CAGE) questionnaire, BMI body mass index, BSMAS Bergen Social Media Addiction Scale, MDDI Muscle Dysmorphic Disorder Inventory, EAT-26 Eating Attitudes Test-26, BSI-GSI Brief Symptom Inventory-Global severity Index

*Number of individual who reported that the most relevantly use psychoactive substance in the previous years was one of the following: cannabis, cocaine, heroin, hallucinogens, amphetamines, tranquillizers, other substances different from alcohol, nicotine and caffeine

**63 T score on the GSI or in two primary BSI dimensions

$p<0.001)$ and BMI $(\rho=-0.164 ; p<0.001)$. Detailed correlations among the variables are reported in Table 2. Differences between individuals with and without PSMU are reported in Table 3.

Results of the mediational model are reported in Table 4 and graphically showed in Fig. 1. Specifically, the total effect was positive and significant $\left(R^{2}=0.11\right.$; $\left.F_{13 ; 707}=6.45 ; p<0.001\right)$, indicating that higher SMArelated symptoms were associated to higher ED-related symptoms $(B=0.213 ; p=0.022)$. Moreover, the effect of SMA-related symptoms on MD-related symptoms was positive and significant $(B=0.136 ; p=0.010)$. In turn, MD-related symptoms were positively related to EDrelated symptoms $(B=0.611 ; p<0.001)$. Lastly, (i) the 
Table 2 Values of Spearman's $\rho$ correlation coefficient among variables in all samples $(N=721)$
23

$3 \quad 4$

$4+5$

$5 \quad 6$

6

7

1. BSMAS total score -

2. MDDI total score

3. EAT-26 total score

4. BSI-GSI

$0.138 * * *$

$0.179 * * *$

$0.430 * * *$

$0.459 * * *$

5. CAGE total score

$0.105^{* *}$

$0.354 * * *$

$0.220 * * *$

$0.382 * * *$

$0.180 * * *$

$-0.163 * * *$

$-0.068$

$-0.090 *$

$0.205 * * *$

6. Age

$-0.164 * * * \quad 0.048$

$-0.011$

$-0.145^{* * *}$

$-0.023$

$-0.116^{* *}$

0.028

$0.165^{* * *} \quad-$

Significant correlations are indicated by asterisk $\left(^{*}\right)$

BSMAS Bergen Social Media Addiction Scale, MDDI Muscle Dysmorphic Disorder Inventory, EAT-26 Eating Attitudes Test-26, BSI-GSI Brief Symptom Inventory-Global severity Index, BMI body mass index, $C A G E$ Cut-Annoyed-Guilty-Eye (CAGE) questionnaire

$* p<0.05 ; * *<<0.01 ; * * * p<0.001$

Table 3 Differences between participants with and without problematic social media use according BSMAS $\geq 19$

\begin{tabular}{|c|c|c|c|c|c|}
\hline Variables & $\begin{array}{l}\text { PSMU- } \\
N=567\end{array}$ & $\begin{array}{l}\text { PSMU+ } \\
N=154\end{array}$ & Test & $p$ & Effect size, $r$ \\
\hline Younger (18-24) young adults, $N(\%)$ & $335(59.1 \%)$ & $106(68.8 \%)$ & $\chi^{2}=4.845$ & 0.028 & 0.082 \\
\hline Age, $\mathrm{M} \pm \mathrm{SD}$ & $24.33 \pm 3.75$ & $23.40 \pm 3.42$ & $U=37260.0$ & 0.005 & 0.104 \\
\hline Females, $N(\%)$ & $382(67.4 \%)$ & $122(79.2 \%)$ & $\chi^{2}=8.081$ & 0.004 & 0.106 \\
\hline Students, $N(\%)$ & $365(64.4 \%)$ & $114(74.0 \%)$ & $\chi^{2}=5.060$ & 0.024 & 0.084 \\
\hline Employed, $N(\%)$ & $166(29.3 \%)$ & $29(18.8 \%)$ & $\chi^{2}=6.687$ & 0.010 & 0.096 \\
\hline Educational level > 13 years, $N(\%)$ & $261(46.0 \%)$ & $55(35.7 \%)$ & $\chi^{2}=5.237$ & 0.022 & 0.085 \\
\hline $\mathrm{BMI}<18.5 \mathrm{~kg} / \mathrm{m}^{2}, N(\%)$ & $53(9.3 \%)$ & $24(15.6 \%)$ & $\chi^{2}=7.065$ & 0.070 & 0.172 \\
\hline BMI between 18.50 and $24.99 \mathrm{~kg} / \mathrm{m}^{2}, N(\%)$ & $422(74.4 \%)$ & $112(72.7 \%)$ & & & \\
\hline BMI between 25 and $29.9 \mathrm{~kg} / \mathrm{m}^{2}, N(\%)$ & $78(13.8 \%)$ & $17(11.0 \%)$ & & & \\
\hline $\mathrm{BMI} \geq 30 \mathrm{~kg} / \mathrm{m}^{2}, N(\%)$ & $14(2.5 \%)$ & $1(0.6 \%)$ & & & \\
\hline $\mathrm{BMI}, \mathrm{M} \pm \mathrm{SD}$ & $22.30 \pm 3.30$ & $21.39 \pm 3.13$ & $U=36207.0$ & $<\mathbf{0 . 0 0 1}$ & 0.121 \\
\hline Married or living with partner, $N(\%)$ & $71(12.5 \%)$ & $13(8.4 \%)$ & $\chi^{2}=1.959$ & 0.162 & 0.052 \\
\hline Tobacco use (last 12 months), $N(\%)$ & $253(44.6 \%)$ & $58(37.7 \%)$ & $\chi^{2}=2.391$ & 0.122 & 0.058 \\
\hline Substances use (last 12 months), $N(\%)$ & $135(22.8 \%)$ & $26(16.9 \%)$ & $\chi^{2}=3.350$ & 0.067 & 0.068 \\
\hline $\mathrm{CAGE} \geq 2, N(\%)$ & $70(12.3 \%)$ & $24(15.6 \%)$ & $\chi^{2}=1.120$ & 0.290 & 0.039 \\
\hline CAGE total, $\mathrm{M} \pm \mathrm{SD}$ & $0.42 \pm 0.82$ & $0.58 \pm 0.99$ & $U=40225.5$ & 0.053 & 0.056 \\
\hline EAT- $26 \geq 20, N(\%)$ & $81(14.3 \%)$ & $39(25.3 \%)$ & $\chi^{2}=10.638$ & 0.001 & 0.121 \\
\hline EAT-26 total score, $\mathrm{M} \pm \mathrm{SD}$ & $9.15 \pm 12.23$ & $13.49 \pm 14.77$ & $U=36653.5$ & 0.002 & 0.114 \\
\hline MDDI > 39, $N(\%)$ & $18(3.2 \%)$ & $8(5.2 \%)$ & $\chi^{2}=1.422$ & 0.233 & 0.044 \\
\hline MDDI total score, $\mathrm{M} \pm \mathrm{SD}$ & $22.92 \pm 7.47$ & $24.84 \pm 8.13$ & $U=37611.0$ & 0.008 & 0.098 \\
\hline BSI cut-off, $N(\%)$ & $99(17.5 \%)$ & $71(46.1 \%)$ & $\chi^{2}=55.143$ & $<\mathbf{0 . 0 0 1}$ & 0.277 \\
\hline BSI-GSI, $M \pm S D$ & $0.84 \pm 0.63$ & $1.45 \pm 0.71$ & $U=21901.5$ & $<0.001$ & 0.354 \\
\hline
\end{tabular}

Bold values represent $p<0.05$

Original, BSMAS Bergen Social Media Addiction Scale, PSMU problematic social media use, CAGE Cut-Annoyed-Guilty-Eye (CAGE) questionnaire, MDDI Muscle Dysmorphic Disorder Inventory, EAT-26 Eating Attitudes Test-26, BSI-GSI Brief Symptom Inventory-Global severity Index

indirect effect was significant $[B=0.083, \mathrm{SE}=0.036$, $p=0.021$ (CI 0.016; 0.159)], thus confirming our mediation hypothesis, and (ii) the direct effect of SMA-related symptoms on ED-related symptoms was not significant
$(B=0.130 ; p=0.139)$, thus suggesting that the association between SMA-related symptoms and ED-related symptoms was mediated by MD-related symptoms. 
Table 4 Results of the mediation models

\begin{tabular}{|c|c|c|c|c|c|c|c|c|c|}
\hline & \multirow{2}{*}{\multicolumn{3}{|c|}{$\frac{\text { Muscle dysmorphia severity }}{\text { Model } 1 \text { (path } a \text { ) }}$}} & \multicolumn{6}{|c|}{ Eating disorder-related symptoms } \\
\hline & & & & \multicolumn{3}{|c|}{ Model 2 (path $c^{\prime}$ ) } & \multicolumn{3}{|c|}{ Model 3 (path $c$ ) } \\
\hline & $B$ & SE & $95 \% \mathrm{CI}$ & $B$ & SE & $95 \% \mathrm{CI}$ & $B$ & SE & $95 \% \mathrm{CI}$ \\
\hline $\begin{array}{l}\text { BSMAS total } \\
\text { score }\end{array}$ & 0.136* & 0.052 & {$[0.033 ; 0.239]$} & 0.130 & 0.088 & {$[-0.042 ; 0.301]$} & $0.213 *$ & 0.093 & {$[0.031 ; 0.395]$} \\
\hline MDDI total score & - & - & - & $\mathbf{0 . 6 1 1} * * *$ & 0.063 & {$[0.488 ; 0.733]$} & - & - & - \\
\hline Age & -0.025 & 0.099 & {$[-0.219 ; 0.169]$} & -0.056 & 0.164 & {$[-0.379 ; 0.266]$} & -0.072 & 0.175 & {$[-0.415 ; 0.271]$} \\
\hline Gender & $-4.152 * * *$ & 0.612 & $\begin{array}{l}{[-5.354} \\
-2.950]\end{array}$ & $3.988 * * *$ & 1.050 & {$[1.926 ; 6.049]$} & 1.453 & 1.083 & {$[-0.674 ; 3.579]$} \\
\hline Student & 1.620 & 1.100 & {$[-0.539 ; 3.778]$} & -0.664 & 1.831 & {$[-4.258 ; 2.930]$} & 0.325 & 1.946 & {$[-3.496 ; 4.145]$} \\
\hline Employed & 0.852 & 1.162 & {$[-1.429 ; 3.132]$} & -0.918 & 1.931 & {$[-4.710 ; 2.874]$} & -0.398 & 2.055 & {$[-4.434 ; 3.637]$} \\
\hline $\begin{array}{l}\text { Educational } \\
\text { level }>13 \text { years }\end{array}$ & 1.102 & 0.604 & {$[-0.084 ; 2.288]$} & 0.051 & 1.006 & {$[-1.924 ; 2.027]$} & 0.724 & 1.069 & {$[-1.374 ; 2.823]$} \\
\hline Marital status & -0.528 & 0.851 & {$[-2.198 ; 1.143]$} & 0.385 & 1.414 & {$[-2.391 ; 3.162]$} & 0.063 & 1.505 & {$[-2.892 ; 3.019]$} \\
\hline $\begin{array}{l}\text { Self-reported } \\
\text { BMI }\end{array}$ & -0.010 & 0.084 & {$[-0.176 ; 0.155]$} & 0.076 & 0.140 & {$[-0.199 ; 0.352]$} & 0.070 & 0.149 & {$[-0.223 ; 0.363]$} \\
\hline Tobacco use & 0.387 & 0.539 & {$[-0.671 ; 1.444]$} & 0.187 & 0.896 & {$[-1.571 ; 1.945]$} & 0.423 & 0.953 & {$[-1.448 ; 2.294]$} \\
\hline $\mathrm{CAGE} \geq 2$ & $2.515 * *$ & 0.800 & {$[0.945 ; 4.085]$} & 1.495 & 1.339 & {$[-1.133 ; 4.123]$} & $3.030 *$ & 1.415 & {$[0.252 ; 5.809]$} \\
\hline Substances use & $2.226 * *$ & 0.683 & {$[0.885 ; 3.568]$} & 0.454 & 1.144 & {$[-1.792 ; 2.700]$} & 1.813 & 1.209 & {$[-0.561 ; 4.187]$} \\
\hline BSI cut-off & $5.024 * * *$ & 0.664 & {$[3.720 ; 6.328]$} & $4.122 * * *$ & 1.148 & {$[1.869 ; 6.375]$} & $7.190 * * *$ & 1.175 & {$[4.882 ; 9.497]$} \\
\hline $\begin{array}{l}\text { Compilation } \\
\text { modality }\end{array}$ & -0.209 & 0.619 & {$[-1.425 ; 1.006]$} & 1.456 & 1.029 & {$[-0.565 ; 3.476]$} & 1.328 & 1.096 & {$[-0.823 ; 3.479]$} \\
\hline \multicolumn{4}{|c|}{$R^{2}=0.18 ; F_{13 ; 707}=12.29 ; p<0.001$} & \multicolumn{3}{|c|}{$R^{2}=0.21 ; F_{14 ; 706}=13.61 ; p<0.001$} & \multicolumn{3}{|c|}{$R^{2}=0.11 ; F_{13 ; 707}=6.45 ; p<0.001$} \\
\hline
\end{tabular}

Coding systems: gender: $1=$ male $2=$ female. Student: $0=$ no $1=$ yes. Employed: $0=$ no $1=$ yes. Educational level $>13$ years: $0=$ no $1=$ yes Marital status: $1=$ unmarried $2=$ married or living with partner. Tobacco use $=0=$ no $1=$ yes. CAGE $\geq 2: 0=$ no $1=$ yes. Illicit drug use: $0=$ no $1=$ yes. BSI cut-off: $0=$ no $1=$ yes. Compilation modality: $1=$ paper/pencil questionnaires $2=$ online questionnaires

Bold values represent $p<0.05$

Original, BSMAS Bergen Social Media Addiction Scale, MDDI Muscle Dysmorphic Disorder Inventory, BMI body mass index, CAGE CutAnnoyed-Guilty-Eye (CAGE) questionnaire, BSI Brief Symptom Inventory

$* p<0.05 ; * * p<0.01 ; * * * p<0.001$

Fig. 1 Graphical representation of the results from the mediation model. The reported estimates were obtained controlling for potentially competing factors (i.e., compilation modality, socio-demographic variables, tobacco use, problematic alcohol use, substances use, self-reported body mass index, and clinically relevant psychopathological distress). BSMAS The Bergen Social Media Addiction Scale, EAT-26 Eating Attitudes Test, $M D D I$ Muscle Dysmorphia Disorder Inventory

\section{Model summary: $\mathrm{R}-\mathrm{Squared}=0.11 ; \mathrm{F}_{13 ; 707}=6.45 ; p<0.001$}

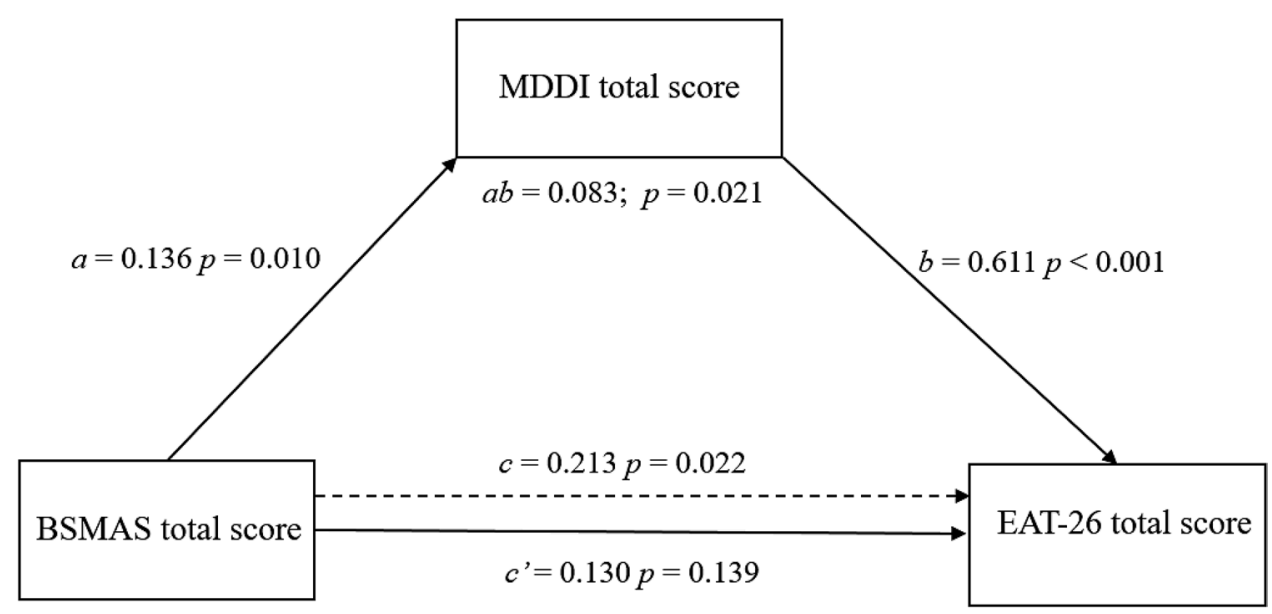




\section{Discussion}

The present cross-sectional study on 721 young adults showed that (i) SMA-related symptoms were significantly associated with both MD- and ED-related symptoms, (ii) the relationship between symptoms of SMA and EDs was mediated by the severity of MD-related symptoms, with relevant confounding factors (sex, age, educational level, marital status, job status, BMI, problematic alcohol use, tobacco use, illicit drug use, psychopathological distress, compilation modality) being controlled for (Table 4; Fig. 1). In addition to such primary findings related to the mediational model, the study provided evidence suggesting that the severity of SMA-related symptoms was significantly directly correlated with overall psychopathological distress and alcohol misuse severity, and significantly inversely correlated with age and BMI (Table 2).

At the clinical level, the present findings contribute to raise awareness on the risks for mental health related to excessive use of SM [58]. According to established cut-off scores (described above) in the assessment measures, in the present sample there were 154 subjects $(21.4 \%)$ who met the criteria for PSMU. Among such individuals, there was, compared to subjects without PSMU (Table 3), a significantly higher proportion of females, of students, of subjects with lower educational level, of younger people, and of unemployed individuals; further, there were lower mean BMI values, more severe MD-related symptoms, and a significantly higher proportion of subjects with clinically relevant psychopathological distress and ED-related symptoms. Subsequently, it is possible that people who experience high degree of SMA (with symptoms related to salience, mood modification, tolerance, withdrawal, conflict, and relapse [35]) may take advantage of specifically tailored interventions focused on the prevention of ED-, MD-, and BDD-related disturbances. Such interventions could potentially include SM-based approaches, as studies have suggested that, in certain circumstances, SM platforms can facilitate social interactions, peer support, and access to care, and can be used to promote mental health awareness, thus providing possible benefits in relation to mental disorders [59, 60]. Further, our results suggest that dysfunctional SM use can be relevant in the etiology and/ or maintenance of ED- and MD-related disturbances, and subsequently clinicians should consider to assess and treat (see for example [61]) SMA-related symptoms in individuals with such conditions.

In relation to MD, the present study suggests that preoccupation over body image and physical activity, with symptoms related to drive for size (e.g., thoughts of being thinner, desire to increase size and strength), appearance intolerance (e.g., negative beliefs about the body), and functional impairment (e.g., excessive and compulsive exercise) [42], is associated with a range of disturbances related to EDs SMA, and psychopathological distress (Tables 2, 4; Fig. 1). Such findings contribute to elucidate the potential risks associated with maladaptive approaches towards sport and exercise. Physical activity is considered as beneficial for health and mental health [62, 63]; however, in the context of disturbances related to body image such as EDs, BDD and $\mathrm{MD}$, exercising may in some cases be part of dysfunctional attitudes and behaviors, and it is, thus, relevant that clinicians as well as people in the field (e.g., athletes, coaches, gym users, trainers) are aware of such potential harms.

\section{What is already known on this subject?}

These results are consistent with evidence suggesting that excessive exposure to SM is associated with ED pathology, and that the preoccupation over physical appearance and muscularity, which can be triggered by certain SM contents (e.g., "thinspiration" and "fitspiration" posts), plays a relevant role in such phenomenon [5, 18-23, 25].

\section{What this study adds?}

The present cross-sectional study on young adults showed (i) that SMA-related symptoms were significantly associated with both MD- and ED-related symptoms, and (ii) that the relationship between symptoms of SMA and EDs was mediated by the severity of MD-related symptoms, with relevant confounding factors being controlled for (Table 4; Fig. 1).

Such findings add specificity to this field of research as the data of the present research have been collected through quantitative measures, while several previous studies on the topic used qualitative approaches. Further, the present research was specifically focused on psychopathological underpinnings related to SMA, and not to PUI in general. This is especially relevant as within the PUI construc, there are a wide range of phenomena markedly different from SM use, such as video gaming, pornography, cybersex, and online gambling [4]. Consistently with such heterogeneity, while in the present study, it was showed that symptoms related to SMA are related to preoccupation over physical appearance/muscularity, ED-related disturbances, and low BMI, previous studies suggested that other aspects of PUI (e.g., videogaming) can in some cases facilitate sedentarism, use of junk food and increased BMI [5, 64]. Thus, given the design of the study, the study findings are specific for SMA, and do not apply to different types of PUI.

\section{Strength and limits}

The present study has several limitations, among which: (i) despite we used an hypothesis-driven statistical approach 
specifically designed to infer causal relationships between associated variables, definitive causality cannot be established as a complete study of mediation processes would require a longitudinal approach, while we used a cross-sectional approach; (ii) self-reports, non-validated by a clinical interview, have been used, whose results may be affected by several biases (e.g., social desirability bias, response bias) [65]; (iii) we did not collect data on the psychiatric history of participants, although the research was specifically directed towards a general population sample and psychopathology was assessed through self-report measures; (iv) a selection bias of the sample may have occurred, i.e., questionnaires might have been more accessible to certain groups of individuals (e.g., students compared to employed or unemployed people, females compared to males); (v) although we assessed the severity of symptoms related to SMA (related to the domains of salience, mood modification, tolerance, withdrawal, conflict, and relapse), we did not perform a qualitative analysis of the SM contents accessed by users; (vi) the amount of subjects who met the criteria for PSMU (21.4\%), clinically relevant ED-related symptoms (16.6\%), clinically relevant MD (3.6\%), clinically relevant psychopathological distress $(23.6 \%)$, and PAU (13.0\%) can be influenced by the fact that the majority of individuals (63.1\%) were recruited during the COVID-19 outbreak, which is a risk factor for increased psychopathology and addiction-related problems [53-55], although the compilation timing (i.e., November 2019-March 2020 vs September-December 2020) was included as a covariate in the mediational model; (vii) although sex was included as a covariate in the mediational model, it is relevant to observe that MD-related symptoms are more frequently observed in males than in females [66], while our sample included more females (69.9\%) than males (30.1\%). Among the strengths of the study: (i) this is, to the best of our knowledge, the first research specifically aimed at investigating in young adults the mediating role of MD-related symptoms on the relationship between SMA- and ED-related symptoms; (ii) the sample size $(n=721)$ was adequate, as indicated by a priori power analysis; (iii) we used extensively validated assessment instruments; (iv) we statistically controlled for potential confounders.

\section{Conclusions}

In conclusions, our findings provide pieces of insight on the complex relationships between SMA, MD, and EDs. It is possible that, while SM use is not detrimental for mental health per se and it can even be integrated in telepsychiatry approaches [59,60], a dysfunctional use of SM can expose users to unrealistic body image ideals; this can have a role in worsening emotional wellbeing and in favoring a maladaptive approach towards physical exercise and food habits, thus contributing to induce in vulnerable subjects symptoms related to MD and EDs. Given the diffusion of SM use among young people, mental health preventive interventions should be encouraged in relation to such phenomena.

Acknowledgements Authors would like to acknowledge the contribution of the "European Network for Problematic Usage of the Internet" (CA16207) funded by the European Cooperation in Science and Technology (COST) in intellectually supporting the study.

Author contributions Study concept and design: CI, FSB, OC, LT, MP, BF, MB; acquisition of subjects and/or data: CI, FSB, GAC, EP, $\mathrm{CM}, \mathrm{LB}, \mathrm{IT}$; analysis and interpretation of the data: CI, FSB, AP, GAC, $\mathrm{EP}, \mathrm{CM}, \mathrm{LB}$, IT; preparation of the manuscript: CI, FSB, AP, OC, LT, $\mathrm{MP}, \mathrm{BF}, \mathrm{MB}$.

Funding Open access funding provided by Università degli Studi di Roma La Sapienza within the CRUI-CARE Agreement. External funding was not involved in the study.

Data availability The data that support the findings of this study are available from the corresponding author upon reasonable request.

\section{Declarations}

Conflict of interest All authors have no current or past potential conflict of interest to disclose, including any financial, personal or other relationships with people or organizations that could inappropriately have influenced the results presented in this paper.

Ethical approval The study was approved by the Ethics committee of the Department of Human Neurosciences of Sapienza University of Rome and was performed in accordance with the Helsinki Declaration criteria.

Consent to participate and to publication All subjects gave their written informed consent to take part to the study and to have the results of the study published in scientific articles.

Open Access This article is licensed under a Creative Commons Attribution 4.0 International License, which permits use, sharing, adaptation, distribution and reproduction in any medium or format, as long as you give appropriate credit to the original author(s) and the source, provide a link to the Creative Commons licence, and indicate if changes were made. The images or other third party material in this article are included in the article's Creative Commons licence, unless indicated otherwise in a credit line to the material. If material is not included in the article's Creative Commons licence and your intended use is not permitted by statutory regulation or exceeds the permitted use, you will need to obtain permission directly from the copyright holder. To view a copy of this licence, visit http://creativecommons.org/licenses/by/4.0/.

\section{References}

1. Mihajlov M, Vejmelka L (2017) Internet addiction: a review of the first twenty years. Psychiatr Danub 29(3):260-272. https://doi. org/10.24869/psyd.2017.260

2. Aboujaoude E (2010) Problematic internet use: an overview. World Psychiatry 9(2):85-90. https://doi.org/10.1002/j.20515545.2010.tb00278.x

3. Tripathi A (2017) Impact of internet addiction on mental health: an integrative therapy is needed. Integr Med Int 4(4):215-222 
4. Fineberg NA, Demetrovics Z, Stein DJ, Ioannidis K, Potenza MN, Grunblatt E et al (2018) Manifesto for a European research network into problematic usage of the internet. Eur Neuropsychopharmacol 28(11):1232-1246. https://doi.org/10.1016/j.euroneuro. 2018.08.004

5. Hinojo-Lucena FJ, Aznar-Diaz I, Caceres-Reche MP, TrujilloTorres JM, Romero-Rodriguez JM (2019) Problematic internet use as a predictor of eating disorders in students: a systematic review and meta-analysis study. Nutrients. https://doi.org/10.3390/ nu11092151

6. Treasure J, Duarte TA, Schmidt U (2020) Eating disorders. Lancet 395(10227):899-911. https://doi.org/10.1016/S0140-6736(20) 30059-3

7. (2013) Diagnostic and statistical manual of mental disorders, 5th edn. American Psychiatric Association, Washington, DC

8. Galmiche M, Dechelotte P, Lambert G, Tavolacci MP (2019) Prevalence of eating disorders over the 2000-2018 period: a systematic literature review. Am J Clin Nutr 109(5):1402-1413. https://doi. org/10.1093/ajen/nqy342

9. Taylor CB, Bryson S, Luce KH, Cunning D, Doyle AC, Abascal $\mathrm{LB}$ et al (2006) Prevention of eating disorders in at-risk collegeage women. Arch Gen Psychiatry 63(8):881-888. https://doi.org/ 10.1001/archpsyc.63.8.881

10. Harrer M, Adam SH, Messner EM, Baumeister H, Cuijpers P, Bruffaerts R et al (2020) Prevention of eating disorders at universities: a systematic review and meta-analysis. Int J Eat Disord 53(6):813-833. https://doi.org/10.1002/eat.23224

11. Tavolacci MP, Dechelotte P, Ladner J (2020) Eating disorders among college students in France: characteristics, help-and care-seeking. Int J Environ Res Public Health. https://doi.org/ 10.3390/ijerph 17165914

12. Jahrami H, Sater M, Abdulla A, Faris MA, AlAnsari A (2019) Eating disorders risk among medical students: a global systematic review and meta-analysis. Eat Weight Disord 24(3):397410. https://doi.org/10.1007/s40519-018-0516-z

13. Fursland A, Watson HJ (2014) Eating disorders: a hidden phenomenon in outpatient mental health? Int J Eat Disord 47(4):422-425. https://doi.org/10.1002/eat.22205

14. Liu CY, Tseng MC, Chen KY, Chang CH, Liao SC, Chen HC (2015) Sex difference in using the SCOFF questionnaire to identify eating disorder patients at a psychiatric outpatient clinic. Compr Psychiatry 57:160-166. https://doi.org/10.1016/j.compp sych.2014.11.014

15. Fornaro M, Daray FM, Hunter F, Anastasia A, Stubbs B, De Berardis D et al (2021) The prevalence, odds and predictors of lifespan comorbid eating disorder among people with a primary diagnosis of bipolar disorders, and vice-versa: systematic review and meta-analysis. J Affect Disord 280(Pt A):409-431. https://doi.org/10.1016/j.jad.2020.11.015

16. Cuzzolaro M (2018) Body dysmorphic disorder and muscle dysmorphia. In: Cuzzolaro M, Fassino S (eds) Body image, eating, and weight. Springer, pp 67-84

17. Brand M, Young KS, Laier C, Wolfling K, Potenza MN (2016) Integrating psychological and neurobiological considerations regarding the development and maintenance of specific Internetuse disorders: an Interaction of Person-Affect-Cognition-Execution (I-PACE) model. Neurosci Biobehav Rev 71:252-266. https://doi.org/10.1016/j.neubiorev.2016.08.033

18. Griffiths S, Castle D, Cunningham M, Murray SB, Bastian B, Barlow FK (2018) How does exposure to thinspiration and fitspiration relate to symptom severity among individuals with eating disorders? Evaluation of a proposed model. Body Image 27:187-195. https://doi.org/10.1016/j.bodyim.2018.10.002

19. Talbot CV, Gavin J, van Steen T, Morey Y (2017) A content analysis of thinspiration, fitspiration, and bonespiration imagery on social media. J Eat Disord 5:40. https://doi.org/10.1186/ s40337-017-0170-2

20. Wick MR, Harriger JA (2018) A content analysis of thinspiration images and text posts on Tumblr. Body Image 24:13-16. https://doi.org/10.1016/j.bodyim.2017.11.005

21. Tiggemann $M$, Zaccardo $M$ (2015) "Exercise to be fit, not skinny": the effect of fitspiration imagery on women's body image. Body Image 15:61-67. https://doi.org/10.1016/j.bodyim. 2015.06.003

22. Prichard I, McLachlan AC, Lavis T, Tiggemann M (2018) The impact of different forms of \#fitspiration imagery on body image, mood, and self-objectification among young women. Sex Roles 78:789-798

23. Robinson L, Prichard I, Nikolaidis A, Drummond C, Drummond M, Tiggemann M (2017) Idealised media images: the effect of fitspiration imagery on body satisfaction and exercise behaviour. Body Image 22:65-71. https://doi.org/10.1016/j.bodyim.2017.06. 001

24. Ioannidis K, Hook RW, Grant JE, Czabanowska K, RomanUrrestarazu A, Chamberlain SR (2021) Eating disorders with over-exercise: a cross-sectional analysis of the mediational role of problematic usage of the internet in young people. J Psychiatr Res 132:215-222. https://doi.org/10.1016/j.jpsychires.2020.11. 004

25. Cataldo I, De Luca I, Giorgetti V, Cicconcelli D, Bersani FS, Imperatori $C$ et al (2021) Fitspiration on social media: body-image and other psychopathological risks among young adults. A narrative review. Emerg Trends Drugs Addict Health 1: 100010. https:// doi.org/10.1016/j.etdah.2021.100010

26. Ryding FC, Kuss DJ (2020) The use of social networking sites, body image dissatisfaction, and body dysmorphic disorder: a systematic review of psychological research. Psychol Pop Media 9(4):412-435

27. Cooper M, Eddy KT, Thomas JJ, Franko DL, Carron-Arthur B, Keshishian AC et al (2020) Muscle dysmorphia: a systematic and meta-analytic review of the literature to assess diagnostic validity. Int J Eat Disord 53(10):1583-1604. https://doi.org/10.1002/eat. 23349

28. Fritz MS, Mackinnon DP (2007) Required sample size to detect the mediated effect. Psychol Sci 18(3):233-239. https://doi.org/ 10.1111/j.1467-9280.2007.01882.x

29. Schou Andreassen C, Billieux J, Griffiths MD, Kuss DJ, Demetrovics $Z$, Mazzoni E et al (2016) The relationship between addictive use of social media and video games and symptoms of psychiatric disorders: a large-scale cross-sectional study. Psychol Addict Behav 30(2):252-262. https://doi.org/10.1037/adb0000160

30. Garner DM, Olmsted MP, Bohr Y, Garfinkel PE (1982) The eating attitudes test: psychometric features and clinical correlates. Psychol Med 12(4):871-878. https://doi.org/10.1017/s003329170 0049163

31. Hildebrandt T, Langenbucher J, Schlundt DG (2004) Muscularity concerns among men: development of attitudinal and perceptual measures. Body Image 1(2):169-181. https://doi.org/10.1016/j. bodyim.2004.01.001

32. Derogatis LR, Melisaratos N (1983) The Brief Symptom Inventory: an introductory report. Psychol Med 13(3):595-605. https:// doi.org/10.1017/S0033291700048017

33. Agabio R, Marras P, Gessa GL, Carpiniello B (2007) Alcohol use disorders, and at-risk drinking in patients affected by a mood disorder, in Cagliari, Italy: sensitivity and specificity of different questionnaires. Alcohol Alcohol 42(6):575-581. https://doi.org/ 10.1093/alcalc/agm072

34. Banyai F, Zsila A, Kiraly O, Maraz A, Elekes Z, Griffiths MD et al (2017) Problematic social media use: results from a largescale nationally representative adolescent sample. PLoS ONE 12(1):e0169839. https://doi.org/10.1371/journal.pone.0169839 
35. Monacis L, de Palo V, Griffiths MD, Sinatra M (2017) Social networking addiction, attachment style, and validation of the Italian version of the Bergen Social Media Addiction Scale. J Behav Addict 6(2):178-186. https://doi.org/10.1556/2006.6.2017.023

36. Gleaves DH, Pearson CA, Ambwani S, Morey LC (2014) Measuring eating disorder attitudes and behaviors: a reliability generalization study. J Eat Disord 2:6. https://doi.org/10.1186/ 2050-2974-2-6

37. Garfinkel PE, Newman A (2001) The eating attitudes test: twentyfive years later. Eat Weight Disord 6(1):1-24. https://doi.org/10. 1007/BF03339747

38. Dotti A, Lazzari R (1998) Validation and reliability of the Italian EAT-26. Eat Weight Disord 3(4):188-194. https://doi.org/10. 1007/BF03340009

39. Rubio-Aparicio M, Badenes-Ribera L, Sánchez-Meca J, Fabris MA, Longobardi C (2020) A reliability generalization meta-analysis of self-report measures of muscle dysmorphia. Clin Psychol Sci Pract 27(1):e12303. https://doi.org/10.1111/cpsp.12303

40. Zeeck A, Welter V, Alatas H, Hildebrandt T, Lahmann C, Hartmann A (2018) Muscle Dysmorphic Disorder Inventory (MDDI): validation of a German version with a focus on gender. PLoS ONE 13(11):e0207535. https://doi.org/10.1371/journal.pone.0207535

41. Longobardi C, Prino LE, Fabris MA, Settanni M (2017) Muscle dysmorphia and psychopathology: findings from an Italian sample of male bodybuilders. Psychiatry Res 256:231-236. https://doi. org/10.1016/j.psychres.2017.06.065

42. Santarnecchi E, Dettore D (2012) Muscle dysmorphia in different degrees of bodybuilding activities: validation of the Italian version of Muscle Dysmorphia Disorder Inventory and Bodybuilder Image Grid. Body Image 9(3):396-403. https://doi.org/10.1016/j. bodyim.2012.03.006

43. Derogatis L (1977) The SCL-90-R manual. Clinical Psychometric Research Unit. Johns Hopkins University School of Medicine, Baltimore, MD

44. Grassi L, Caruso R, Mitchell AJ, Sabato S, Nanni MG (2018) Screening for emotional disorders in patients with cancer using the Brief Symptom Inventory (BSI) and the BSI-18 versus a standardized psychiatric interview (the World Health Organization Composite International Diagnostic Interview). Cancer 124(11):2415-2426. https://doi.org/10.1002/cncr.31340

45. Grassi L, Rasconi G, Pedriali A, Corridoni A, Bevilacqua M (2000) Social support and psychological distress in primary care attenders. Ferrara SIMG Group. Psychother Psychosom 69(2):95100. https://doi.org/10.1159/000012372

46. Grassi L, Mondardini D, Pavanati M, Sighinolfi L, Serra A, Ghinelli F (2001) Suicide probability and psychological morbidity secondary to HIV infection: a control study of HIV-seropositive, hepatitis $\mathrm{C}$ virus (HCV)-seropositive and HIV/HCV-seronegative injecting drug users. J Affect Disord 64(2-3):195-202. https://doi. org/10.1016/s0165-0327(00)00244-5

47. De Leo D, Frisoni GB, Rozzini R, Trabucchi M (1993) Italian community norms for the Brief Symptom Inventory in the elderly. Br J Clin Psychol 32(Pt 2):209-213. https://doi.org/10.1111/j. 2044-8260.1993.tb01045

48. Dhalla S, Kopec JA (2007) The CAGE questionnaire for alcohol misuse: a review of reliability and validity studies. Clin Investig Med 30(1):33-41. https://doi.org/10.25011/cim.v30i1.447

49. Imperatori C, Corazza O, Panno A, Rinaldi R, Pasquini M, Farina B et al (2020) Mentalization impairment is associated with problematic alcohol use in a sample of young adults: a cross-sectional study. Int J Environ Res Public Health. https://doi.org/10.3390/ ijerph17228664

50. Fritz CO, Morris PE, Richler JJ (2012) Effect size estimates: current use, calculations, and interpretation. J Exp Psychol Gen 141(1):2-18. https://doi.org/10.1037/a0024338
51. Rosenthal R, DiMatteo MR (2001) Meta-analysis: recent developments in quantitative methods for literature reviews. Annu Rev Psychol 52:59-82. https://doi.org/10.1146/annurev.psych.52.1.59

52. Hayes AF (2012) PROCESS: a versatile computational tool for observed variable mediation, moderation, and conditional process modeling. [White paper], pp 1-39. http://www.afhayes.com/public/process2012.pdf

53. Hossain MM, Tasnim S, Sultana A, Faizah F, Mazumder H, Zou L et al (2020) Epidemiology of mental health problems in COVID19: a review. F1000Res 9:636. https://doi.org/10.12688/f1000 research.24457.1

54. Panno A, Carbone GA, Massullo C, Farina B, Imperatori C (2020) COVID-19 related distress is associated with alcohol problems, social media and food addiction symptoms: insights from the Italian experience during the lockdown. Front Psychiatry 11:577135. https://doi.org/10.3389/fpsyt.2020.577135

55. Jiang Y (2021) Problematic social media usage and anxiety among university students during the COVID-19 pandemic: the mediating role of psychological capital and the moderating role of academic burnout. Front Psychol 12:612007. https://doi.org/10.3389/ fpsyg.2021.612007

56. Salthouse TA (2011) All data collection and analysis methods have limitations: reply to Rabbitt (2011) and Raz and Lindenberger (2011). Psychol Bull 137(5):796-799. https://doi.org/10. 1037/a0024843

57. Baron RM, Kenny DA (1986) The moderator-mediator variable distinction in social psychological research: conceptual, strategic, and statistical considerations. J Personal Soc Psychol 51(6):11731182. https://doi.org/10.1037/0022-3514.51.6.1173

58. Cataldo I, Lepri L, Yee Neoh MJ, Esposito G (2021) Social media usage and development of psychiatric disorders in childhood and adolescence: a review. Front Psychiatry

59. Naslund JA, Bondre A, Torous J, Aschbrenner KA (2020) Social media and mental health: benefits, risks, and opportunities for research and practice. J Technol Behav Sci 5:245-257

60. Latha K, Meena KS, Pravitha MR, Dasgupta M, Chaturvedi SK (2020) Effective use of social media platforms for promotion of mental health awareness. J Educ Health Promot 9:124. https://doi. org/10.4103/jehp.jehp_90_20

61. Hou Y, Xiong D, Jiang T, Song L, Wang Q (2019) Social media addiction: its impact, mediation, and intervention. Cyberpsychol J Psychosoc Res Cyberspace 13(1):article 4

62. Rebar AL, Stanton R, Geard D, Short C, Duncan MJ, Vandelanotte C (2015) A meta-meta-analysis of the effect of physical activity on depression and anxiety in non-clinical adult populations. Health Psychol Rev 9(3):366-378. https://doi.org/10.1080/ 17437199.2015.1022901

63. Love R, Adams J, van Sluijs EMF, Foster C, Humphreys D (2018) A cumulative meta-analysis of the effects of individual physical activity interventions targeting healthy adults. Obes Rev 19(8):1164-1172. https://doi.org/10.1111/obr.12690

64. Kracht CL, Joseph ED, Staiano AE (2020) Video games, obesity, and children. Curr Obes Rep 9(1):1-14. https://doi.org/10.1007/ s13679-020-00368-z

65. Demetriou C, Ozer BU, Essau CA (2015) Self-report questionnaires. In: Cautin RL, Lilienfeld SO (eds) The encyclopedia of clinical psychology. John Wiley \& Sons

66. American Psychiatric Association (2013) Diagnostic and statistical manual of mental disorders, 5th edn. American Psychiatric Association, p 245

Publisher's Note Springer Nature remains neutral with regard to jurisdictional claims in published maps and institutional affiliations. 